STICHTING

MATHEMATISCH CENTRUM

2e BOERHAAVESTRAAT 49

AMSTERDAM

ZW 1956-002

A simple method of calculating

electrostatic capacity

Prof.dr. C.J. Bouwkamp

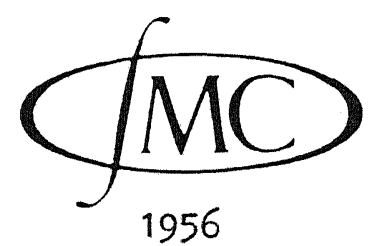


Voordracht door Prof.Dr C.J. Bouwkamp voor het Mathematisch Centrum op 27 januari 1956:

\section{A SIMPIE METHOD OF CALCULATING ELECTROSTATIC CAPACITY}

\section{ABSTRACT}

Attention is drawn to a simple theorem enabling the evaluation of the electrostatic capacity of certain conductors in free space such as a system of two spheres in contact with each other or a "ring without hole".

\section{INTRODUCTION}

It is well known that from the solution of certain problems in electrostatics we may obtain the solution of others by means of a transformation by reciprocal radii (inversion in a sphere, Kelvin transformation). The purpose of this note is to draw attention to a simple application of the general theory of images by means of which it is easy to determine the electrostatic capacity of certain conducting surfaces with respect to infinity. Despite the simple nature of the underlying theorem, to be proved below, I have not been able to trace it in the available litera.. ture. In addition, oral communication with various persons conversant with potential theory convinced me that the theorem is not known very well if at all.

\section{THEOREM}

Let $C$ denote the capacity with respect to infinity of a bounded conductor with surface $S$ in free space. Let $R$ denote any sphere with center $M$ and radius $a_{\text {, and let }} S^{\prime}$ be the image of $S$ with respect to $R$ in the sense of inversion by reciprocal radii. Then $C=a^{2} V_{0}$, in which $V_{0}$ is the potential at $M$ due to the charges induced on the conductor $S$ ' by a negative unit point charge located at $M$ when $S^{\prime}$ is connected to earth.

\section{Proof of the theorem}

Let us introduce spherical polar coordinates around the center $M$ of the sphere $R$. Functions occurring in the sequal will depend on the radial distance $r$ as well as on the angular variables. However, to simplify the notation, we shall omit any explicit reference to the angular variables. Note that in the case of inversion with respect to $R$ the field point and its image have the same angular variables, while the product of their radial distances equals $a^{2}$. 
Let $V(r)$ be the solution of Laplace's equation in the space exterior to $S$ assuming unit value on $S$ and vanishing at infinity. That is, $V(r)$ is the potential outside $S$ if $S$ is kept at unit potential. Now the capacity of the conductor $S$ is easily seen to be the coefficient of $r^{-1}$ in the expansion of $\mathrm{V}$ according to inverse powers of $r$, viz.

$$
V(r)=C r^{-1}+O\left(r^{-2}\right), r \rightarrow \infty
$$

From Kelvin's theory it is further known that the function

$$
V \cdot(r) \equiv r^{-1} V\left(a^{2} r^{-1}\right)
$$

Is also a solution of Laplace's equation, and it is so to that side of $S$ ' which contains $M$ as an inner point ( $M$ is the image of the point at infinity under the KeIvin transformation (2)).

Now the value of $V(r)$ on the surface $S$ and the value of $V\left(a^{2} r^{-1}\right)$ on the surface $S^{\prime}$ are exactly the same, both being unity all over $S$ and $S^{\prime}$. As a consequence, $V^{\prime}$ equals $\mathrm{r}^{-1}$ at all points of $\mathrm{S}^{\prime}$. If we then add to $\mathrm{V}^{\prime}$ the potential due to a negative unit point charge placed at the origin $M$, we get the harmonic function

$$
V^{\prime \prime}(r)=-r^{-1}+r^{-1} V\left(a^{2} r^{-1}\right)
$$

which obviously vanishes on the surface $S^{\prime}$. Therefore $V^{\prime \prime}$ is the potential due to a negative unit point charge located at $M$ in the presence of the conductor $S^{\prime}$ when the latter is connected to earth.

The second term of the right-hand side of equation (3) is the potential of the charges induced on $S^{\prime}$ by the point charge at $M$. Its value at this very point $M$ is equal to

$$
\begin{aligned}
V_{0} & =\lim _{r \rightarrow 0} r^{-1} V\left(a^{2} r^{-1}\right) \\
& =\lim _{r \rightarrow \infty} a^{-2} r V(r)=a^{-2} C,
\end{aligned}
$$

in virtue of equation (1). Hence $c=a^{2} V_{0}$, which completes the proof of our theorem.

\section{REMARK}

It is illustrative to verify the theorem if $S$ is a sphere by taking $R$ arbitrarily. The most simple case is if $S$ and $R$ are taken identical; then $S^{\prime}$ also coincides with $S$ and $R$. The case of two mutually orthogonalIy intersecting spheres is also easy to deal with, but the result is too well known to require any further examination. Below are given two neat 
examples that, although not claimed to be new, are less known.

EXAMPLE NO.1

Consider the system of two spheres in contact with each other (radii a and $\underline{b} ; a<b$, say). To calculate the capacity $\mathrm{C}_{a b}$ of this conductor, we apply our theorem with $\mathrm{R}$ being the sphere of radius $2 \mathrm{~b}$ with center at the point of contact The image $S^{\prime}$ then is a system of two parallel planes at distances $p=2 b$ and $a=2 b^{2} a^{-1}$ respectively from the origin $M$ and at opposite sides of it. Put the two planes at zero potential and place a negative unit point charge at $M$. The field between the two planes is easy to find by the method of images. If $V_{0}$ is the value of the secondary potential at the locus of the point charge, then

$$
\begin{aligned}
& V_{0}=(2 p)^{-1}-(2 p+2 q)^{-1}+(4 p+2 q)^{-1}-(4 p+4 q)^{-1}+(6 p+4 q)^{-1}-(6 p+6 q)^{-1}+\ldots \\
& \text { + similar terms with } p \text { and } q \text { interchanged } \\
& =2 / 2 p(2 p+2 q)+2 q /(4 p+2 q)(4 p+4 q)+2 q /(6 p+4 q)(6 p+6 q)+\ldots \\
& \text { + similar terms with } p \text { and } q \text { interchanged } \\
& =\sum_{n=1}^{\infty} 2 q /[2 n p+(2 n-2) q][2 n p+2 n q]+\text { similar terms }
\end{aligned}
$$$$
=\frac{1}{2} q(p+q)^{-2} \sum_{n=1}^{\infty} \frac{1}{n\left(n-\frac{q}{p+q}\right)}+\text { similar terms. }
$$

The remaining infinite series can be expressed in terms of the logarithmic derivative of the gamma function, viz.

$$
\psi(-z)=-\gamma+z^{-1}-z \sum_{n=1}^{\infty} \frac{1}{n(n-z)},
$$

where $\gamma$ is Euler's constant. Using some transformation properties of the psi-function, we find that

$$
\begin{aligned}
V_{0} & =\frac{1}{2}(p+q)^{-1}\left\{2-2 \gamma+p / q+q / p-\Psi\left(-\frac{p}{p+q}\right)-\Psi\left(-\frac{p}{p+q}\right)\right\} \\
& =-\frac{1}{2}(p+q)^{-1}\left\{2 \gamma+\psi\left(\frac{p}{p+q}\right)+\Psi\left(\frac{p}{p+q}\right)\right\} .
\end{aligned}
$$

Finally, recaliing that $\mathrm{C}_{\mathrm{ab}}=(2 \mathrm{~b})^{2} \mathrm{~V}_{\mathrm{o}}$ and inserting the values of $\mathrm{p}$ and q, we find for the capacity

$$
c_{a b}=-\frac{a b}{a+b}\left\{2 \gamma+\psi\left(\frac{a}{a+b}\right)+\psi\left(\frac{b}{a+b}\right)\right\} \text {. }
$$


Two special cases of this general formula can be eas1ly checked. If we let $a$ tend to zero, and recall that in this limiting case $\Psi\left(\frac{a}{a+b}\right) \sim-b / a$, we flnd $\mathrm{C}_{\mathrm{ab}} \rightarrow \mathrm{b}$ as $1 \mathrm{t}$ should. Further, In the case of two equal spheres $(a=b)$ it 1 s found that

$$
c_{\text {aa }}=2 a \log 2
$$

It may be worth while to repeat the argument in this case of two equal spheres, because then the series can be summed in an elementary manner, in view of

$$
1082=1-1 / 2+1 / 3-1 / 4+\ldots \cdot
$$

Deta1ls, however, are lept to the reader.

\section{EXAMPLE NO.?}

Let us next consider the conductor obtained by rotating a circle around one of its tangents; it is a "ring without hole". Let a be the radius of the c1rcle, and let $M$ be the center of the ring. To apply our theorem. we take $R$ to be the sphere of radius $2 a$ with center at $M$. Inversion of the ring in $\mathrm{R}$ leads to a cylinder with circular cross-section of radivs 2a. The capacity of the ring is $\mathrm{C}_{\text {ring }}=4 \mathrm{a}^{2} \mathrm{~V}$, in which $\mathrm{V}_{0}$ is the value of the secondary potential at $M$ when the cylinder wall is connected to earth and a negative unit point charge is placed at $M$ on the axis of the cylinder. The solution of the latter potential problem is known. For details the reader is referred to a jolnt paper by N.G. de Bruijr. and the author entitled "The Electrostatic Field of a point charge Inside a Cylinder, in Connection with Wave Gulde Theory", which appeared in Journal of Applied Physies 18 (1947) 562-577. From equation (17) of that paper the secondary potential inside the cylinder (radius $2 a$ ) is found to be

$$
V_{0}(r)=+\frac{2}{\pi} \int_{0}^{\infty} \frac{K_{0}(2 a \lambda)}{I_{0}(2 a \lambda)} I_{0}(\rho \lambda) \cos z \lambda d \lambda,
$$

in which $\rho$ and $Z$ are cylindrical coordinates. Consequently

$$
c_{r i n g}=\frac{4 a}{\pi} \int_{0}^{\infty} \frac{K_{0}(t)}{I_{0}(t)} d t \text {, }
$$

In which $K$ and I are modifled Bessel functions in the notation of Watson. An alternative expression is found by integration by parts (see equation 26 of the paper cited);

$$
c_{\text {ring }}=\frac{4 a}{\pi} \int_{\delta}^{\infty} \frac{d t}{I_{0}^{2}(t)} .
$$


In the mentioned paper the integral was evaluated to be

$$
\frac{1}{\pi} \int_{0}^{\infty} \frac{d t}{I_{0}^{2}(t)}=c .4353382 .
$$

If $\supset$ is the maximum diameter $(D=4 a)$ of the ring without hole, we fin for the capacity

$$
C_{\text {ring }}=0.4353382 \mathrm{D}
$$

\section{CONCLUDING REMARKS}

The result of equation (7) is not new. As a matter of fact, I accldent ally traced the formula in a British journal which perhaps appeared the end of the last century. Unfortunately I forgot the name of the author who was a well-known mathematiclan. If I remember well, this samo author dealt also with a heart-shaped conductor of revolution which appeared to be the image of a parabolold of revolution with respect to a sphere with center at the focus. The calculation of the capacity of this shape of conductor can be done by our theorem as well, and the result is quite similar to that of equation (7) with its typical Bescel function integral. As a matter cf fact, the known result for the capacity of the ring as given by the unidentified British mathematicia and the results of N.G. de Bruijn and myself as mentioned above led mr to discover the theorem of this paper.

It is perhaps interesting, in view of recent work on isoperimetric in. equalities by Polya and others, to express the capacity of the ring $1 .$. terms of the ratio volume/surface area of the ring. The volume of the ring without hole is $2 \pi^{2} a^{3}$, and its surface area is $4 \pi^{2} a^{2}$, which un. the ratio equal to $\frac{1}{2}$ a. Accordingly

$$
c_{\text {ring }}=3.4827 \frac{\text { volume }}{\text { surface area }} \text {. }
$$

For a sphere the numerical factor is 3 . 